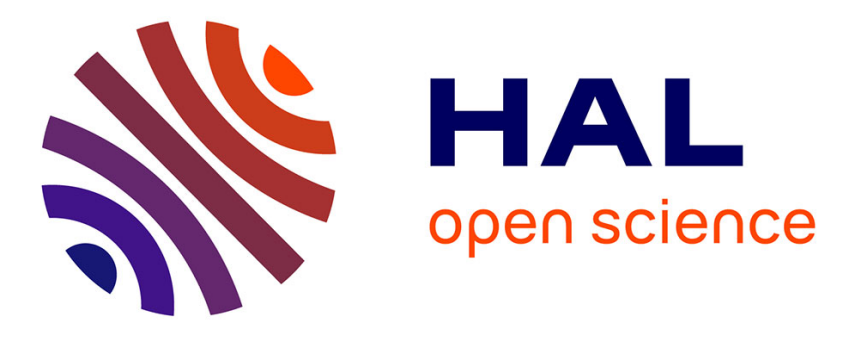

\title{
Exploring fusion at extreme sub barrier energies with weakly bound nuclei
}

A. Shrivastava, A. Navin, A. Lemasson, K. Ramachandran, V. Nanal, M.

Rejmund, K. Hagino, T. Ichikawa, S. Bhattacharyya, Avhishek Chatterjee, et al.

\section{To cite this version:}

A. Shrivastava, A. Navin, A. Lemasson, K. Ramachandran, V. Nanal, et al.. Exploring fusion at extreme sub barrier energies with weakly bound nuclei. Physical Review Letters, 2009, 103, pp.232702. 10.1103/PhysRevLett.103.232702 . in2p3-00436084

\section{HAL Id: in2p3-00436084 https://hal.in2p3.fr/in2p3-00436084}

Submitted on 26 Nov 2009

HAL is a multi-disciplinary open access archive for the deposit and dissemination of scientific research documents, whether they are published or not. The documents may come from teaching and research institutions in France or abroad, or from public or private research centers.
L'archive ouverte pluridisciplinaire HAL, est destinée au dépôt et à la diffusion de documents scientifiques de niveau recherche, publiés ou non, émanant des établissements d'enseignement et de recherche français ou étrangers, des laboratoires publics ou privés. 


\title{
Exploring fusion at extreme sub barrier energies with weakly bound nuclei
}

\author{
A. Shrivastava, ${ }^{1,2, *}$ A. Navin, ${ }^{2}$ A. Lemasson, ${ }^{2}$ K. Ramachandran,${ }^{1}$ V. Nanal,${ }^{3}$ \\ M. Rejmund ${ }^{2}$ K. Hagino, ${ }^{4}$ T. Ichikawa, ${ }^{5}$ S. Bhattacharyya, ${ }^{6}$ A. Chatterjee, ${ }^{1}$ \\ S. Kailas, ${ }^{1}$ K. Mahata, ${ }^{1}$ V.V. Parkar, ${ }^{1,3}$ R.G. Pillay, ${ }^{3}$ and P.C. Rout ${ }^{1}$ \\ ${ }^{1}$ Nuclear Physics Division, Bhabha Atomic Research Centre, Mumbai 400085, India \\ ${ }^{2}$ GANIL, CEA/DSM - CNRS/IN2P3, Bd Henri Becquerel, BP 55027, F-14076 Caen Cedex 5, France \\ ${ }^{3}$ DNAP, Tata Institute of Fundamental Research, Mumbai 400005, India \\ ${ }^{4}$ Department of Physics, Tohuku University, Sendai 980-8578, Japan \\ ${ }^{5}$ Yukawa Institute for Theoretical Physics, Kyoto University, Kyoto 606-8502, Japan \\ ${ }^{6}$ Variable Energy Cyclotron Centre, 1/AF Bidhan Nagar, Kolkata 700064, India
}

\begin{abstract}
Results of measurement of residues formed in fusion of ${ }^{6} \mathrm{Li}$ with ${ }^{198} \mathrm{Pt}$ in the energy range of $0.68<\mathrm{E} / \mathrm{V}_{b}<1.3$ using a new sensitive off-beam technique are reported. The fusion excitation function and the derived average angular momenta do not indicate a change of slope at deep sub-barrier energies, contrary to recent observations. The present results for a system with weakly bound projectile confront the current understanding of the fusion hindrance at these low energies, underlying the role of internal re-organization on the dynamical path towards fusion.
\end{abstract}

PACS numbers: 25.70.Jj, 25.70.-z, 25.60.Je

Nuclear reactions around the Coulomb barrier are a vast reservoir for signatures of various aspects of basic quantum mechanics. The tunneling phenomena, in particular, can be probed under diverse conditions ranging from the effect of dissipation in heavy systems to the role of pairing. These unique features, in addition to the effects of inter-connectivity of intrinsic properties in the entrance channel on different processes, provide insights into various quantum mechanical effects [1]. In the last few years improved sensitivity in the measurements have challenged the understanding of the mechanism of tunneling through multidimensional barriers. The latest addition to this artillery is the study of the isospin degree of freedom and the effect of weak binding, which can be probed using recently available radioactive-ion beams and loosely bound stable projectiles.

Recent measurements with medium-heavy nuclei highlighted the change of slope of the fusion excitation function at deep sub-barrier energies compared to coupled channels calculations [2]. The energy where these deviations begin, referred to as the threshold energy for observing fusion hindrance, has been parametrized and its implications on the fusion with light nuclei of astrophysical relevance have been discussed [3]. Dasso and Pollarolo [4] pointed out that the cross-sections at deep sub-barrier energies could be used as an unique tool to obtain the value of the nuclear potential at small distances (see also [5]). More recently Ichikawa et al. showed that the potential energy at the touching point strongly correlates with this threshold energy [6]. Mişicu and Esbensen proposed a potential with a shallow pocket (as compared to that obtained from Woods-Saxon parametrization) based on a sudden approximation, where the reaction takes place so rapidly that the colliding nuclei overlap with each other without changing their density [7]. A repulsive core included to take into account the nuclear com- pressibility arising due to Pauli exclusion principle, modifies the depth and the shape of the minima of the internuclear potential at small distances. They also showed that, depending on the choice of the couplings used in the calculations, there were surprising structures in the calculated average angular momentum at these low energies [7]. The nucleus-nucleus interaction potentials extracted from the microscopic time-dependent HartreeFock theory indicate that at low energies the frozen density approximation breaks down implying re-organization of the internal degrees of freedom [8]. Based on an adiabatic picture, a dynamical two-step model was proposed by Ichikawa et al. to explain the deep sub-barrier fusion data [9]. It should be noted that the above two approaches based on the sudden and adiabatic models predict different angular momentum distributions [10]. The measurement of the average angular momentum could also discriminate between the two approaches mentioned above $[7,9]$ that describe the fusion data equally well. In the sudden approach, using a shallow potential [7], the average angular momentum of the compound nucleus is always smaller than in the two-step adiabatic model [9] at low energies.

The fusion of weakly bound nuclei, which is a subject of current interest, has yet not been investigated at energies far below the barrier. For exotic weakly bound projectiles, a fully quantum mechanical time dependent wave-packet approach using a three body model also predicts a suppression of total fusion compared to corresponding stable nuclei over the entire energy range [11]. Experimental studies at deep sub-barrier energies have been restricted mainly to the measurement of fusion cross-sections of symmetric systems with the exception of ${ }^{16} \mathrm{O}+{ }^{204,208} \mathrm{~Pb}$ systems, spanning a range of "stiffness", reduced mass and Q-values [2, 3, 12-14]. Hence measurements of fusion cross-sections at low energies for a 


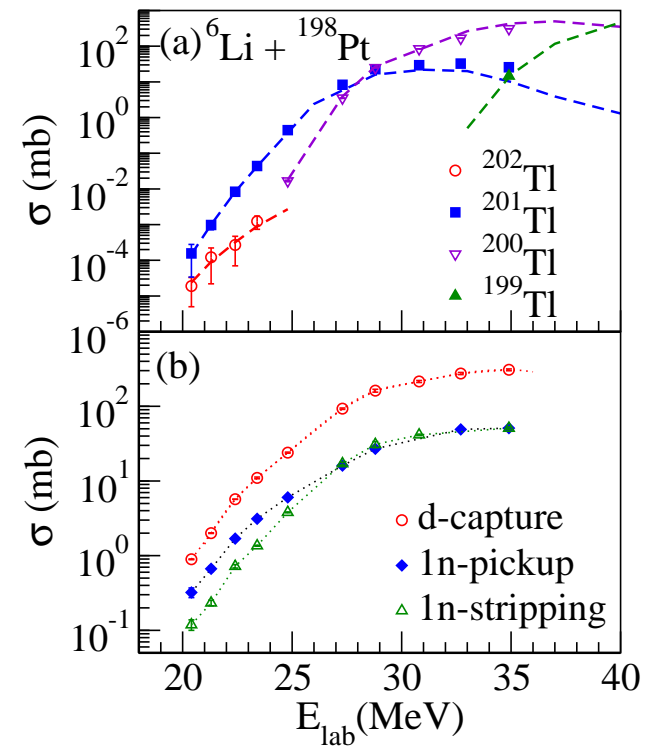

FIG. 1: (color online) Excitation functions for ${ }^{6} \mathrm{Li}+{ }^{198} \mathrm{Pt}$ system (a) Evaporation residues from compound nuclear fusion. Dashed curves are results of statistical model calculations (see text). (b) Cross-sections of residues arising from $d$-capture $\left({ }^{198-200} \mathrm{Au}\right), 1 \mathrm{n}$-pickup $\left({ }^{197} \mathrm{Pt}\right)$ and $1 \mathrm{n}$-stripping $\left({ }^{199} \mathrm{Pt}\right)$ reactions. The dashed lines are to guide the eye.

completely different entrance channel are necessary to understand the tunneling process at energies well below the barrier.

In this work, we present a fusion measurement at deep sub-barrier energies for studying the phenomenon of fusion hindrance, in case of the weakly bound projectile ${ }^{6} \mathrm{Li}\left(\mathrm{S}_{\alpha / t}=1.45 \mathrm{MeV}\right)$. The present system incidentally also has a positive $\mathrm{Q}$-value $(8.5 \mathrm{MeV})$ for the formation of the compound nucleus which is the case for only two recently studied systems $[13,14]$ at these low energies. A new sensitive off-beam- $\gamma$-spectroscopy method to obtain the cross-section of residues from fusion, utilizing a coincidence between characteristic KX-rays and $\gamma$-rays from the daughter nuclei, has been used [15]. This coincidence measurement permitted the accurate and precise determination of the residue cross-sections by reducing the background. The average angular momenta and the total cross-sections for the associated direct reactions are also presented over the same energy range. Form factors for direct reactions, obtained from peripheral reactions, may not be realistic at shorter distances between the reacting nuclei [16] and the present data, at energies below the barrier will provide constraints to probe such a conjuncture.

The experiment was performed at Pelletron Linac Facility-Mumbai, using beams of ${ }^{6} \mathrm{Li}(5-35 \mathrm{pnA})$ on a ${ }^{198} \mathrm{Pt}$ target in the range of 20 to $35 \mathrm{MeV}$. The targets were self supporting rolled foils of ${ }^{198} \mathrm{Pt}$ (95.7\% enriched, $\sim 1.3 \mathrm{mg} / \mathrm{cm}^{2}$ thick) followed by an $\mathrm{Al}$ catcher foil of thickness $\sim 1 \mathrm{mg} / \mathrm{cm}^{2}$. Fresh targets were used and back-
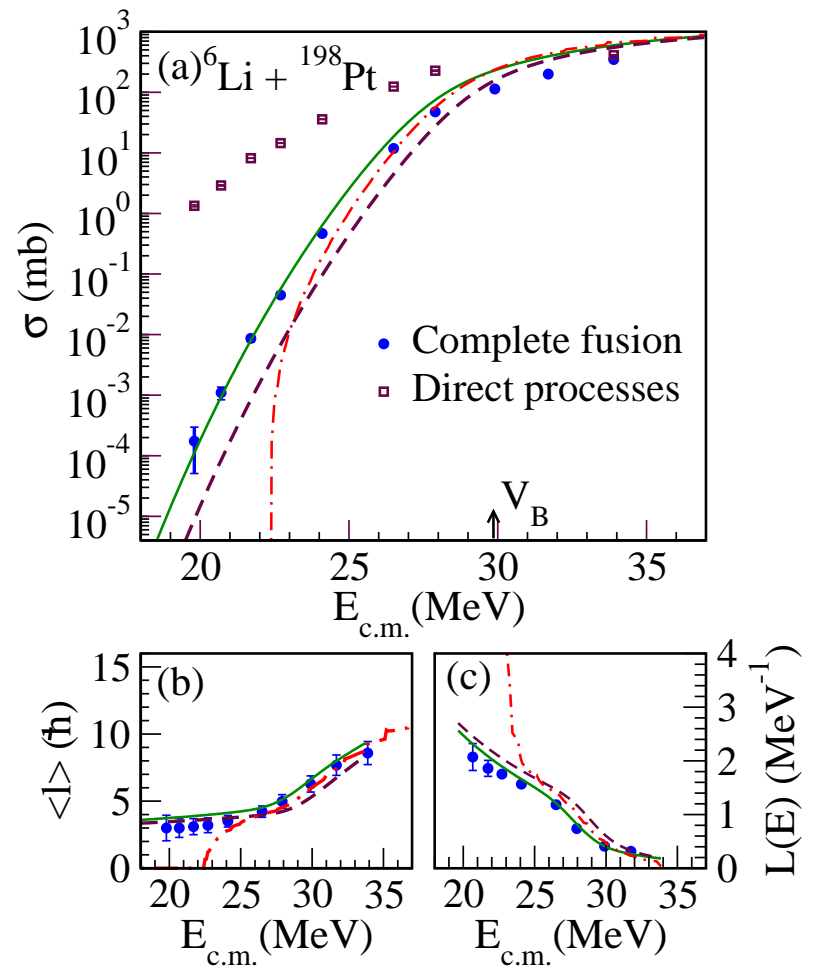

FIG. 2: (color online) Fusion excitation function and derived observables for ${ }^{6} \mathrm{Li}+{ }^{198} \mathrm{Pt}$ system (a) Cross-sections for compound-nucleus formation and direct processes obtained from a sum of the partial cross-sections shown in Fig.1 (a) and (b) respectively. The arrow indicates value of the Coulomb barrier $\left(\mathrm{V}_{B}\right)$. (b) Average angular momentum and (c) Logarithmic derivative of the fusion excitation function. The results of the coupled-channels calculations using the WS potential (solid line) along with single channel calculations using the WS potential (dashed line) and the M3Y potential with a repulsive core (dot-dashed lines) are shown in panels (a)-(c) (see text).

ground data was collected before each irradiation. Two efficiency calibrated HPGe detectors were placed face to face for performing $\mathrm{KX}-\gamma$-ray coincidence of the decay radiations from the irradiated sample. The sample was positioned symmetrically between the two detectors in a close geometry (1.5 $\mathrm{mm}$ from the face of each detector). The measurements were performed in a low background setup with a graded shielding. The reaction products were uniquely identified by means of their characteristic $\gamma$-ray energies and half-lives which in the case of fusion lead to ${ }^{199-202} \mathrm{Tl}$ residues. The $\gamma$-ray yields of the daughter nuclei were extracted by gating on their KX-ray transitions [15]. The resulting cross-sections of the residues are plotted in Fig. 1(a). Due to the increased sensitivity of the KX- $\gamma$-coincidence method, cross-sections down to a few nano-barns could be measured. The estimation of errors for low counting rates was made assuming Poisson statistics and using the method of maximum likelihood [17]. Statistical model calculations for the com- 
pound nuclear decay were performed using PACE4 [18] with the cross-section for each partial wave obtained from coupled-channels calculations. The results from the calculation are displayed as dashed curves in Fig. 1(a) and explain the data rather well. The $\gamma$-ray yields for residues formed after $d$-capture $\left({ }^{198-200} \mathrm{Au}\right)$ and neutron transfer reactions $\left({ }^{197,199} \mathrm{Pt}\right)$ were extracted from inclusive $\gamma$ ray measurements and these cross-sections are plotted in Fig. 1(b). The cross-sections for $d$-capture (incomplete fusion) are larger than those for the neutron transfer at all energies. To the best of our knowledge these are the lowest energies below the barrier where direct reaction cross-sections have been measured.

The fusion cross-sections, obtained from the sum of the measured evaporation residue cross-sections, are plotted in Fig. 2(a) for ${ }^{6} \mathrm{Li}+{ }^{198} \mathrm{Pt}$. Corrections for ${ }^{196} \mathrm{Pt}$ impurity in the target $(2.56 \%)$ were found to be negligible ( $<1 \%$ even at highest energy). The cross-sections for the sum of deuteron-capture and neutron-transfer (plotted as open squares) are larger than those for fusion by orders of magnitude at deep sub-barrier energies. In the present work the average angular momenta $(\langle l\rangle)$ have been derived from the fusion excitation function as suggested in Refs.[19, 20] and are plotted in Fig. 2(b).

Calculations using the coupled-channels (CC) code CCFULL [21] were performed with the ingoing-waveboundary condition. Two sets of calculations, one using a standard Woods-Saxon potential (WS) $\left(\mathrm{V}_{0}=110 \mathrm{MeV}\right.$, $\mathrm{r}_{0}=1.1 \mathrm{fm}$ and $\mathrm{a}=0.63 \mathrm{fm}$ ) and the other based on the M3Y folded potential are presented. The potentials are plotted in Fig. 3. The calculations using the WS potential included the quadrupole excitation in ${ }^{198} \mathrm{Pt}$, considering coupling in the vibrational model. For ${ }^{6} \mathrm{Li}$ the $1^{+}$(ground state) and the unbound $3^{+}$states were assumed to be from a $\mathrm{K}^{\pi}=1^{+}$rotational band. The results of the calculation with and without the inclusion of the couplings are shown in Fig. 2(a). At energies above the barrier the calculations overestimate the data, as expected from earlier studies involving weakly bound nuclei [22]. As can be seen in the figure, the $\mathrm{CC}$ calculations reproduce the data for energies around and well below the barrier. Plotted in Fig. 2(c) is the logarithmic derivative of the fusion crosssection $(\mathrm{L}(\mathrm{E})=\mathrm{d}[\ln (\sigma \mathrm{E})] / \mathrm{dE})$ obtained using three point numerical derivative. This representation provides an alternate way to illustrate any deviations in the slope of the fusion excitation function independent of the weight of the lowest barrier. The $\mathrm{CC}$ calculations reproduce well both the experimental slope $\mathrm{L}(\mathrm{E})$ and the $\langle l\rangle$ values (Fig. 2(b)) over the entire range of energy. Thus for ${ }^{6} \mathrm{Li}$ $+{ }^{198} \mathrm{Pt}$, the CC calculations successfully explain the fusion excitation function along with the average angular momentum consistently, implying absence of the fusion hindrance at deep sub-barrier energies.

The lack of the fusion hindrance observed in the present case from the above calculations is also possible if the threshold value for the onset of fusion hindrance

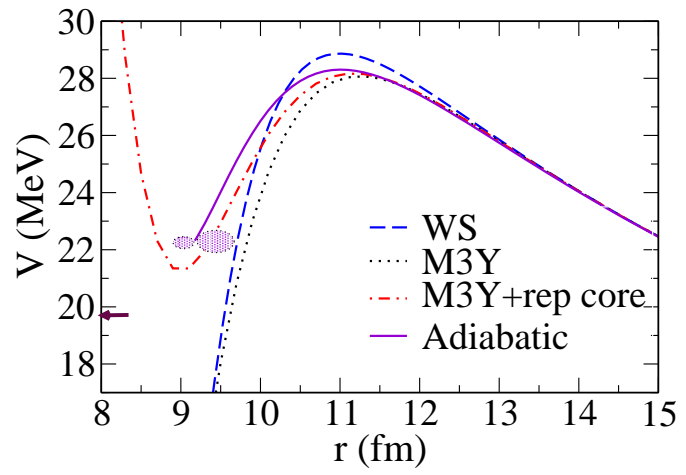

FIG. 3: (color online) Inter-nuclear potentials for ${ }^{6} \mathrm{Li}+{ }^{198} \mathrm{Pt}$ using the WS (long dashed line), the M3Y-double folding (dotted line) and the M3Y with a repulsive core (dasheddotted line). The adiabatic potential is shown as a solid curve up to the formation of a neck-configuration. The arrow indicates the lowest center of mass energy where the fusion cross sections were measured.

was not reached. This does not appear to be the case, as shown below. The threshold energy was computed following two independent approaches. The M3Y potential with repulsive core [7] was calculated taking the density distributions of ${ }^{6} \mathrm{Li}$ and ${ }^{198} \mathrm{Pt}$ from Ref.[23] and for the repulsive core, $\mathrm{V}_{\text {rep }}=570 \mathrm{MeV}$ and $\mathrm{a}_{\text {rep }}=0.35 \mathrm{fm}$ (yielding a value of $\mathrm{K}=234 \mathrm{MeV}$ ) as a representative choice for the parameters. The resulting potential (Fig. 3) has a minimum at 21.3 MeV and as discussed in Refs. [4, 7] the threshold energy is larger than this value. Adopting a smaller value of $\mathrm{a}_{r e p}(=0.3 \mathrm{fm})$ lowers the potential minimum $(=15.6 \mathrm{MeV})$, but such a small value of $\mathrm{a}_{r e p}$ is inconsistent with that for other systems [7]. Alternatively following the two-step adiabatic model of Ichikawa with Krappe-Nix-Sierk potential [9], the energy at the touching configuration, related to the threshold energy is calculated to be $22.3 \mathrm{MeV}$ (Fig. 3). The present measurements extend down to $\mathrm{E}_{c m}=19.8 \mathrm{MeV}$, which is well below the threshold energy computed from both the approaches, although there may be some ambiguity for the definition of the touching point for a weakly bound nucleus.

Single-channel calculations using the above M3Y potential with a repulsive core were also performed as suggested in Ref.[7] and the results are shown in Fig. 2. The calculated fusion cross-sections, for energies lower than $22 \mathrm{MeV}$, fall off steeply and are orders of magnitude lower than the corresponding single channel calculations using the WS potential (Fig.2(a)). The effect of coupling on the calculated fusion cross-sections are found to be small from the $\mathrm{CC}$ calculations as seen in the same figure. A similar behavior was observed in Ref.[22]. Hence at these energies, even after including the effect of coupling the calculated fusion cross-sections using the M3Y+repulsive core potential will be much lower than the measured fusion cross-sections. The calculated $\mathrm{L}(\mathrm{E})$ values also do 
not agree with the data and rise more steeply at low energies (Fig. 2(c)). The corresponding mean angular momentum drops to zero around an energy of $\mathrm{E}=22$ $\mathrm{MeV}$ which is also inconsistent with the experimental data (Fig. 2(b)).

A shallow potential obtained using the M3Y interaction with a repulsive core successfully describes the fusion cross-sections at deep sub-barrier energies for symmetric, asymmetric and positive reaction Q-valued systems $[7,13,16]$. But for the present system with a weakly bound projectile this potential does not reproduce the trend of the fusion excitation function, $\mathrm{L}(\mathrm{E})$ and $\langle l\rangle$. The present results suggest that the inner part of the interaction potential becomes deeper, going from a symmetric to a weakly bound asymmetric system, implying reduced contribution of the repulsive core. A plausible reason for this could be as follows. As the nuclei start overlapping, due to the weak binding of one partner, the Fermi energies of the two interacting nuclei are very different and will tend to equilibrate rather fast. Thus Pauli blocking is expected to be less effective for asymmetric systems involving weakly bound nuclei as compared to symmetric systems [24]. The actual form of the repulsive core is expected to depend also on the extent of the adiabatic nature of the collision [7]. At energies well below the barrier the adiabatic approximation is expected to be more appropriate where nuclear reactions take place following the minimum energy path allowing for the readjustment of the densities as a function of collective variables [8]. The predictions based on the adiabatic model of Ichikawa et al. [10] already appear to give the correct behavior for the average angular momentum in the medium-mass symmetric systems though currently such calculations are not possible for asymmetric systems.

In summary, we have presented the fusion excitation function for a very asymmetric system involving a weakly bound projectile at energies well below the barrier. This study shows the absence of fusion hindrance, pointing to the limitation of the sudden approximation for modeling reactions in such systems. It would be of interest to see whether this arises solely from the effect of weakly bound cluster structure [25] or also due to difference in transition from the sudden to the adiabatic potential. In order to address this question, both the sudden and adiabatic approaches would require extensions by taking into account the weakly bound nature of the projectile nucleus. An independent way to probe this conjuncture would be to analyze data of alpha-induced fusion at deep sub barrier energies, for which measurements presently do not exist. Such data due to both experimental and theoretical simplicity could also provide an ideal testing ground for studying the effects of irreversible environmental couplings on the collision of nuclei [26].

We acknowledge Y. Blumenfeld's help for providing targets, D. Lacroix for stimulating discussions and the accelerator staff for smooth running of the machine.

* email: aradhana@barc.gov.in

[1] N. Keeley et al., Prog. in Part. and Nucl. Phys. 59, 579 (2007); M. Dasgupta et al., Ann. Rev. Nucl. Part. Sci. 48, 401 (1998).

[2] C.L. Jiang et al., Phys. Rev. Lett. 89, 052701 (2002).

[3] C.L. Jiang, K.E. Rehm, B.B. Back and R.V.F. Janssens, Phys. Rev. C 79, 044601 (2009), and references therein.

[4] C. H. Dasso and G. Pollarolo, Phys. Rev. C 68, 054604 (2003).

[5] K. Hagino and Y. Watanabe, Phys. Rev. C 76, 021601(R) (2007).

[6] T. Ichikawa, K. Hagino and A. Iwamoto, Phys. Rev. C 75, 064612 (2007).

[7] S. Mişicu and H. Esbensen, Phys. Rev. Lett. 96, 112701 (2006); ibid. Phys. Rev. C 75, 034606 (2007).

[8] K. Washiyama and D. Lacroix, Phys. Rev. C 78, 024610 (2008); A.S. Umar and V.E. Oberacker, Phys. Rev. C 74, 021601(R) (2006).

[9] T. Ichikawa, K. Hagino and A. Iwamoto, Phys. Rev. C 75, 057603 (2007).

[10] T. Ichikawa, K. Hagino and A. Iwamoto, FUSION08, AIP Conf. Proc. 1098, 32 (2009).

[11] M. Ito et al., Phys. Lett. B 637, 53 (2006).

[12] M. Dasgupta et al., Phys. Rev. Lett. 99, 192701 (2007).

[13] C. L. Jiang et al., Phys. Rev. C 78, 017601 (2008).

[14] A.M. Stefanini et al., Phys. Rev. C 78, 044607 (2008).

[15] A. Lemasson et al., Nucl. Instr. Meth. A 598, 445 (2009).

[16] H. Esbensen and S. Mişicu, Phys. Rev. C 76, 054609 (2007).

[17] W.-M. Yao et al., J. Phys. G 33, 1 (2006).

[18] A. Gavron, Phys. Rev. C 21, 230 (1980).

[19] A.B. Balantekin and P.E. Reimer, Phys. Rev. C 33, 379 (1986).

[20] C.V.K. Baba, Nucl. Phys. A 553, 719c (1993); V. Tripathi et al., Phys. Rev. Lett. 88, 172701 (2002).

[21] K. Hagino et al., Comp. Phys. Comm. 123, 143 (1999).

[22] M. Dasgupta et al., Phys. Rev. C 66, 041602(R) (2002).

[23] L. C. Chamon et al., Phys. Rev. C 66, 014610 (2002).

[24] D. Lacroix, "Macroscopic approaches for fusion reactions", Ecole Joliot-Curie, Maubuisson, France (2002). "http://www.cenbg.in2p3.fr/heberge/EcoleJoliotCurie /coursJC/JOLIOT-CURIE 2002.pdf".

[25] E. Uegaki and Y. Abe, Prog. Theor. Phys. 90, 615 (1993).

[26] A. Diaz-Torres et al., Phys. Rev. C 78, 064604 (2008). 